\title{
Estrategias metodológicas que utilizan los docentes en la enseñanza de la Ley de Ampere bajo el enfoque de Díaz y Hernández
}

Methodological strategies used by teachers in teaching the Ampere Law under the focus of Diaz and Hernández

\begin{tabular}{ll}
\hline Yosnelly Jackeline Muñoz Jurado & $\begin{array}{l}\text { Everth Velez } \\
\text { everthvelez2@gmail.com } \\
\text { yosnelly20@gmail.com }\end{array}$ \\
$\begin{array}{l}\text { Instituto de Educación Activa, } \\
\text { Venezuela }\end{array}$ & Malpica, Venezuela \\
\hline
\end{tabular}

Artículo recibido en junio 2020 | Arbitrado en julio 2020 | Publicado en septiembre 2020

\section{Resumen}

El presente estudio pretendió describir las estrategias metodológicas implementadas por los docentes para la enseñanza del contenido de la Ley de Ampere, a la luz de los estudios realizados por los pedagogos Frida Díaz y Gerardo Hernández, en el circuito escolar $\mathrm{N}^{\circ} 4$ del Municipio Los Guayos del Estado Carabobo, Venezuela. El mismo, se realizó con un enfoque cuantitativo, diseño no experimental, de campo. La población estuvo conformada por un grupo de doce docentes del área de conocimiento de física del municipio escolar antes mencionado, a los cuales se les aplicó un instrumento previamente elaborado y utilizado por Colmenares y Henríquez (2011) en su trabajo de grado. Con los hallazgos obtenidos en la presente investigación, se logró reconocer el grado de profundidad cómo son utilizadas las estrategias metodológicas para la enseñanza de la Ley de Ampere bajo el enfoque de Díaz y Hernández, además los aspectos relevantes.

\footnotetext{
Abstract

This descriptive study aims to describe the methodological strategies implemented by teachers to teach the content of the Ampere Law, in light of the studies carried out by pedagogues Frida Díaz and Gerardo Hernández, in school circuit No. 4 of the Municipality Los Guayos of the Carabobo State, Venezuela. It was carried out with a quantitative approach, a non-experimental, field design. The population was made up of a group of twelve teachers in the area of knowledge of physics from the aforementioned school municipality, to whom an instrument previously developed and used by Colmenares and Henríquez (2011) in their graduate work was applied. With the findings obtained in the present investigation, it was possible to recognize the degree of depth how methodological strategies are used for the teaching of Ampere's Law under the approach of Díaz and Hernández, in addition to the relevant aspects.
}

\section{Palabras clave:}

Estrategias metodológicas; enseñanza; Ley de Ampere

\section{Keywords:}

Methodological strategies; teaching; Ampere's Law 
INTRODUCCIÓN

En la actualidad existen infinidades de estrategias que un docente puede implementar al momento de enseñar algún contenido, y la mayoría persiste en la escasa variedad de recursos sin hacer uso de estrategias significativas al momento de explicar temas de física, especialmente el contenido de Ley de Ampere que requiere necesariamente de la implementación de estrategias para exponer de manera adecuada los fenómenos que estén vinculados con el contenido. Generalmente en todas las instituciones se evidencia las mismas características didácticas para la enseñanza de ciertos contenidos de física.

Actualmente, no es difícil darse cuenta que cada vez son menos los estudiantes que se interesan en esta ciencia, ya que suele ser un contenido al que no le ha atribuido algún valor significativo en las escuelas. Sin embargo, podrían señalarse como principales agentes causantes de esta problemática las estrategias de carácter tradicionalista que subestiman la importancia del aprendizaje significativo. Cabe destacar que la poca comprensión alcanzada en estos temas obliga al estudiantado a desertar las aulas de clase, promoviendo el bajo rendimiento y la escasa matricula en esta área de conocimientos.

Los contenidos de física en la educación secundaria son abordados de manera superficial y muchas veces, los agentes de enseñanza que corren a cargo de la instrucción de los mismos, no cuentan con la documentación suficiente para darlos a conocer de manera que el aprendiz pueda incorporar dichos contenidos a su estructura cognoscitiva, además de poder ubicar estos conceptos como fenómenos que tienen ocurrencia en su medio.

A lo largo de todas las asignaturas enmarcadas en el currículo de la Educación Media, la física y en especial la Ley de Ampere presenta un basamento conceptual muy abstracto y poco intuitivo, por lo que el estudiante a menudo enfrenta dificultades de comprensión que los conducen a sentimientos de frustración.

En la gran mayoría de los institutos educativos, los profesionales de la educación se han dado a la tarea de desempeñar sus actividades con un enfoque tradicionalista, en donde la función de transmisor de conocimientos no da lugar a la interacción recíproca con los miembros del alumnado. En este sentido, los docentes se mantienen al margen de los procesos de adquisición de conocimientos por parte de los estudiantes. Limitándose solo a escribir fórmulas y ecuaciones e insistiendo en la actividad memorística de las mismas, así como también de algunos conceptos particulares, sin que ninguna de estas variedades refleje conexión alguna con la realidad social del estudiante. En síntesis la mayoría de las estrategias implementadas consisten en actividades memorísticas y de repetición que obedecen a patrones preestablecidos por la escuela tradicional. Y en este contexto se han enmarcado muchos de los docentes encargados de las disciplinas como la física, cuyo carácter experimental se ha obviado en todo momento en la praxis docente.

En un breve recorrido por las aulas, donde se imparten los contenidos de esta asignatura se puede apreciar cómo los docentes se concentran casi de manera exclusiva a la transcripción de complicados ejercicios extraídos de manera textual de los libros, con la finalidad de que los alumnos procedan a plasmarlos en sus cuadernos, sin cerciorarse efectivamente del nivel de aprendizaje adquirido por los mismos, ni de los procedimientos empleados para su resolución.

La actividad memorística se emplea demasiado para lograr el aprendizaje de las 
principales definiciones y conceptos propios de los contenidos de la asignatura. Muchas de las teorías y leyes que conforman a la física tienen la particularidad de ser muy abstractos para su eficaz comprensión. Por lo que casi siempre se demanda del estudiante un esfuerzo adicional de su nivel intelectual para lograr la apropiación de estas ideas. Esfuerzo que por demás el principal cuerpo de estudiantes no está dispuesto a realizar, bien sea por factores vinculados a su desmotivación, su bajo interés, y a la falta de conexión de estas ideas con aspectos prácticos de su cotidianidad.

En este orden de ideas se maneja la concepción de la enseñanza de la física como una práctica promotora de aprendizajes errados y confusos. Ya que la gran parte de los alumnos que se enfrentan a estos métodos de enseñanza no se apropian correctamente de los conceptos y definiciones de las leyes físicas por estar más interesados en resolver los ejercicios que les son asignados usando para ello recetas procedimentales que el docente se encarga de transmitir, como una especie de modelo riguroso e inflexible.

Más aun, las estrategias didácticas, concebidas como un recurso variado de implementación por parte de los educadores, se ven limitadas tan solo a la transmisión de contenidos de manera verbal, empleándose como únicos medios de apoyo: el pizarrón y el marcador. El docente promedio de física, en el aula venezolana, no emplea otros medios didácticos para la ejecución de su trabajo. Por ende, el nivel de comprensión que se adquiere por el alumnado, en esta asignatura, es muy bajo y sujeto casi siempre a elementos de confusión.

La eficiente adquisición de los fenómenos electromagnéticos tendrá su auge en la interrelación que existe entre las clases teóricas y las actividades prácticas, en las cuales, más allá de las ecuaciones y leyes, el estudiante pueda interpretar resultados prácticos. Es necesario que los ciudadanos comprendan el enorme papel que tiene el desarrollo de las ciencias en este particular, para que las generaciones de relevo cuenten con el bagaje cultural óptimo para apuntarse también en los muchos avances de los que las generaciones de hoy estamos siendo testigos.

El incentivo educativo juega entonces un rol muy importante en la promoción de estos valores con el objeto de que la actividad científica salga desde muy temprana edad a los fines de dar promoción eficaz, al desarrollo de la ciencia y la cultura. Por otro lado, el aliciente de la cultura científica en cada país nace principalmente del entusiasmo que se le invierta a las disciplinas relacionadas con la ciencia, con motivo de que sus educandos puedan apreciar el potencial tecnológico que pueden aportar dentro del contexto cultural y social de sus realidades particulares.

Las estrategias de enseñanza de las que disponen los docentes en la educación secundaria parecen carecer del incentivo suficiente para que el alumno se sienta motivado a cumplir con las tareas que le son asignadas en un marco de educación tradicional. En este sentido, cabe responder a la interrogante ¿Qué estrategias implementan los docentes para la enseñanza de la ley de Ampere?, a través de del objetivo general: Describir las estrategias metodológicas que utilizan los docentes para la enseñanza de la Ley de Ampere bajo el enfoque de Díaz y Hernández en el circuito $\mathrm{n}^{\circ} 4$ del municipio Los Guayos del estado Carabobo, el cual se logró cumplir con los objetivos específicos siguientes: 1. Determinar las estrategias preinstruccionales usadas por los docentes para la enseñanza de la Ley de Ampere al inicio de la clase, bajo el enfoque de Díaz y Hernández en el circuito $\mathrm{N}^{\circ} 4$ del municipio Los 
Guayos del estado Carabobo; 2. Establecer las estrategias coinstruccionales usadas por los docentes en esta área.; 3. Precisar las estrategias postinstruccionales usadas por los docentes para la enseñanza de la Ley de Ampere en el cierre de la clase, bajo el enfoque de Díaz y Hernández.

La presente investigación permitió conocer la magnitud de alcance de las diversas actividades orientadas a conseguir el aprendizaje significativo de contenidos del área de conocimientos de física, tal como la ley de ampere con el fin de medir su nivel de impacto y efectividad. Las estrategias analizadas en esta investigación son de carácter constructivista, la cual permite la promoción diversos métodos con fines pedagógicos para apoyar la enseñanza de la física, en especial del contenido Ley de Ampere. Asimismo, la descripción de las estrategias analizadas en esta investigación conseguirá que los docentes encargados de su aplicación sean más conscientes al momento de la planificación programática en las que se ven comprometidos en su actividad pedagógica.

Las estrategias metodológicas que aplican los agentes de enseñanza en los diferentes contextos educativos en los cuales interactúan, en realidad, son apoyos que contribuyen a la promoción de aprendizajes y generación de ideas en los aprendices. Es por lo que las mismas constituyen una fuente promisoria de transmisión efectiva del conocimiento. Así mismo, se describen las estrategias metodológicas llevadas a cabo en tan importante labor, al tratar de constituir un puente de conocimiento entre el docente y el educando, con la finalidad de que la comunicación entre ambos se pueda dar en condiciones de reciprocidad y riqueza cultural.

Las alternativas dadas a conocer en este trabajo, también podrían ser de utilidad para la promoción de escenarios de aprendizaje en donde el educando de la educación primaria pueda apreciar la naturaleza de los fenómenos de carácter físico que tienen ocurrencia en su entorno, y así mismo, generar espacios de participación para fomentar la identificación de los fenómenos antes mencionados. La activación del conocimiento en el estudiante pasa también por la observación de modelos educativos con los cuales el docente se apoye para que el estudiante pueda apropiarse del conocimiento a través de la aplicación de estrategias de enseñanza que los alumnos sean capaces de seguir.

Un antecedente relevante para este estudio fue el de Méndez y Villavicencio (2017), en su trabajo titulado: "Enseñanza del electromagnetismo a través de aplicaciones experimentales". Sobre este particular se propuso el uso de actividades basadas en la experimentación que tuvieran estrecha relación con las aplicaciones tecnológicas actuales, en donde se reflejaran muchos de los usos que en la cotidianidad, los alumnos, casi sin saberlo, le dan a los principios básicos inherentes al electromagnetismo. Los resultados de esta investigación arrojan que los alumnos pueden llegar a un mejor entendimiento de estos contenidos haciendo uso de simulaciones computarizadas, y a su vez experimentos sencillos que reflejen en ellos un aprendizaje significativo, permitiéndoles relacionar los resultados de las experiencias con los conocimientos que ya poseen previamente.

Uno de los fundamentos teóricos por los cuales se apoyó el siguiente estudio fue la teoría del aprendizaje significativo desarrollada por Ausubel (2006). En ella, se establece que el aprendizaje puede tener lugar, bien sea por recepción, es decir, por medio de la enseñanza explicativa o exposiciones orales, o por medio, de un aprendizaje significativo, en donde los contenidos que pretenden ser asimilados 
pueden ser adquiridos de manera independiente. En este sentido, según los aportes del citado autor, la función de la psicología educativa en la educación de los profesores se basa en la premisa de que existen principios generales del aprendizaje significativo en el salón de clases que se pueden derivar de una teoría razonable acerca de tal aprendizaje.

Estos principios proporcionarán el fundamento psicológico necesario para que los aspirantes a profesores dispongan de un banco de información pedagógica que les permita advertir los métodos prácticas de enseñanza más idóneos con los diferentes ambientes y condiciones en los que se genera el proceso de aprendizaje escolar. Como también para seleccionar aquellos métodos que serán también más favorables de entre aquellos que sean impuestos desde el magisterio. Todos los métodos de enseñanza requieren estar relacionados con la naturaleza del proceso de aprendizaje y considerar los factores cognoscitivos, afectivos y sociales que lo influyen.

Las dos dimensiones en las que se genera el proceso de aprendizaje son: recepción descubrimiento y repetición aprendizaje. Aunque el término recepción haga referencia con la enseñanza explicativa, y que de acuerdo a lo reseñado por Ausubel (2006), ésta se asociará con la dimensión de repetición aprendizaje, esencialmente, el aprendizaje por recepción y por descubrimiento, pueden ser ambos significativos en tanto se presente las siguientes condiciones: si el estudiante posee la disposición de relacionar el material presentado con su estructura particular de conocimiento, es decir, que dicho material de aprendizaje, lo pueda asociar el estudiante con su grado de conocimiento o su basamento cultural hasta ese momento adquirido. La segunda condición guarda relación con la potencialidad del material, el cual si es sensible, y relacionable con la estructura cognoscitiva particular del estudiante, dicho aprendizaje se pueda dar de manera sustancial y no arbitraria.

En este orden de ideas, el aprendizaje por recepción, según los señalamientos del autor en consideración, el contenido o material de aprendizaje se le presenta al estudiante, y este posteriormente lo internaliza y lo asocia con la estructura cognoscitiva que en este se halle. En tanto que, en el aprendizaje por descubrimiento, la manifestación del conocimiento se efectúa independientemente de una exposición de contenidos, es decir, el estudiante logra descubrir el contenido principal del aprendizaje, lo asimila y luego procede a internalizarlo asociándolo e incorporando a su basamento de ideas y conceptos hasta ese momento concebido.

Las teorías desarrolladas por el autor hacen énfasis en el papel que juega el aprendizaje en el salón de clases, y que el mimo para que sea considerado como significativo tiene que ver con la estructuración lógica a que los términos hacen referencia. El aprendizaje presupone tanto que el estudiante pase una actitud de aprendizaje significativo como que la tarea de aprendizaje sea potencialmente significativa. Para que ambos escenarios se den se requiere que la relación de ideas nuevas representadas simbólicamente se haga de manera sustancial y no arbitraria.

Es decir que las nuevas ideas se puedan relacionar con algún aspecto existente específicamente importante de la estructura cognoscitiva del estudiante. Y que además, la tarea de aprendizaje sea potencialmente significativa, ello implica que la misma sea relacionable con su estructura de conocimiento sobre una base no arbitraria y no al pie de la letra. De esta manera se puede conocer que, si la disposición del estudiante no es del orden de 
una actitud de aprendizaje significativo entonces aunque el material de aprendizaje tenga características de potencialidad hacia el aprendizaje tenga características de potencialidad hacia el aprendizaje significativo, este proceso se dará de manera mecánica, lo cual conlleva a la memorización sin comprensión de significado. Y además, aunque la disposición del estudiante sea significativa, si el material de aprendizaje no es de potencialidad significativa, el proceso de aprendizaje se dará, de igual forma, en condiciones desprovistas de significado.

Desde el punto de vista del desarrollo del aprendizaje escolar, el autor antes señalado establece que ningún interés teórico es más urgente que la necesidad de distinguir los tipos de aprendizaje que tienen lugar en el salón de clases, la manera de reconocer estos aprendizajes es seccionarlos en aprendizajes por recepción y descubrimiento, y el aprendizaje dado a manera de repetición. (Ausubel, 2006)

En el aprendizaje por recepción, el contenido solo se le es presentado al estudiante, y este, luego, internaliza el conocimiento atendiendo el carácter de potencialidad del material presentado, es decir, que las ideas expuestas sean relacionables con la estructura cognoscitiva presente en el estudiante. Por otra parte, en el aprendizaje por descubrimiento, el contenido o material de aprendizaje es descubierto por el estudiante y luego este reorganiza la información para posteriormente integrarla a su cuerpo de conocimiento.

Por lo tanto, el aprendizaje por recepción y descubrimiento son dos formas de aprendizaje diferentes que tienen lugar en un escenario escolar. Sin embargo, es el primero de ellos, el aprendizaje por recepción, el que encuentra un espacio de mayor representación en las aulas de clases.
Se tomaron conceptos e iniciativas de los trabajos de Díaz y Hernández (2002), en donde las estrategias metodológicas que empleadas estuvieron fundamentadas por las referencias hacia la construcción de un aprendizaje significativo, en donde los participantes del proceso enseñanza y aprendizaje, intercambiaran ideas y experiencias con el docente a cargo de la enseñanza.

De acuerdo a la metodología implementada existen enfoques teóricos que conducen a la orientación de las prácticas escolares de la enseñanza, con la finalidad de que estas puedan implicar la asimilación de contenidos sustanciales en los individuos en los cuales se ejecutan. Es por lo que, en este orden de ideas, al darse señalamiento de los métodos y técnicas representativos de una enseñanza sustancial o que favorezcan la promoción y la relación de ideas y de significados concernientes a las materias de estudio, es valorable considerar la aproximación constructivista que está ligada al aprendizaje escolar.

Por lo tanto, el enfoque constructivista en materia educativa, implica la convergencia de distintas corrientes psicológicas que contribuyen a la promoción de aprendizajes en el aula de clases y que atienden problemas como: la identificación y atención oportuna de elementos presentes en la praxis educativa y que son inherentes a los estudiantes que enfrentan este proceso, tales como la motivación, los intereses, y las necesidades; el desarrollo psicológico del individuo, en el plano intelectual y en su interacción con los tipos de aprendizaje que se gestan en el aula de clases; el reconocimiento de diversos tipos de aprendizaje escolar con atención a los componentes intelectuales, afectivos y sociales que guardan relación con los mismos; la búsqueda de 
alternativas novedosas de tratamiento curricular asociadas al diseño de estrategias y métodos de enseñanza que posibiliten el aprendizaje; la concepción constructivista del aprendizaje escolar parte de la idea de que el fin último de la escuela consiste en promover el desarrollo intelectual del estudiante a través de la participación activa del mismo en actividades intencionales, planificadas y sistemáticas que contribuyan a crear en este las aptitudes necesarias para la promoción de los contenidos que se le impartan.

Incluso se ha señalado que mediante la realización del aprendizaje de índole significativo, el educando tiene la posibilidad de alcanzar un desarrollo intelectual, que le permite construir significados que enriquecen su conocimiento del mundo social. En este sentido, los aspectos relevantes en que pueden sustentarse la praxis educativa son: la memorización de carácter comprensivo, los aprendizajes significativos, y la funcionalidad de lo aprendido en el contexto educativo. Además, las posturas constructivistas que indican los autores antes señalados, rechazan la idea de ver en el educando un mero receptor de saberes y consecuentemente un mecánico reproductor de los mismos. El constructivismo asociado a las características de la actividad escolar induce a que las instituciones educativas fomenten en sus educandos la individualización y la socialización pertinentes a la generación de una identidad personal en el marco del contexto social y cultural en el que se desenvuelven estos educandos.

En este orden de ideas, la finalidad de las corrientes constructivistas convergen en el escenario en donde los individuos sean capaces de realizar por si solos, procesos de aprendizaje significativo, cuya naturaleza ejemplifique el acto de pensar y actuar contendidos significativos y contextuales.

El principio de las estrategias metodológicas para la enseñanza parte también de considerar el significado del papel que juega el enseñante en un escenario en donde se promueva el proceso continuo y dinámico de enseñanza aprendizaje. En primer lugar la idea de la enseñanza está relacionada con el conjunto de premisas de las que se apoya el maestro para construir un fundamento o cimiento sobre el cual se puedan erigir posesos de aprendizaje. A esto supone también la variabilidad característica a que está sujeta el acto educativo en el contexto institucional, como también por la naturaleza y características propias de los educandos, en lo referente al contexto educativo, para que se dé lugar de manera provechosa el proceso de enseñanza, se atienden, en algún orden, las disposiciones y objetivos que direcciona el aspecto curricular del sistema. En lo concerniente al rol que juegan los educandos, las variables que afectan el proceso de enseñanza tienen que ver con los elementos cognoscitivos, afectivos y sociales, que están siempre presentes en los mismos, y que de acuerdo al grupo, representan una realidad única digna de ser estimada, antes de dar inicio a cualquier actividad o proceso de enseñanza.

En el seno de las realidades escolares todo proceso de enseñanza implica relaciones de reciprocidad entre los acores que intervienen en dicho proceso, por parte de los docentes, estos tienen la opción de generar una comunicación efectiva en condiciones favorables para la transmisión de contenidos e ideas. Dado que la enseñanza también implica la necesidad de poner en consideración todas 
estas ideas, para que esta pueda ser efectiva requiere esencialmente la comprensión de que, las realidades en los escenarios escolares son distintas una de la otra, en virtud de los muchos fenómenos psicológicos, sociales, culturales e instruccionales que se involucran en el proceso, por lo que, cada realidad escolar es única e irrepetible.

Con base en lo anterior, se concluye que las estrategias utilizadas, aunque bien pensadas y organizadas, tienen que diferir en sus aplicaciones para atender efectivamente a todas estas realidades. No existe, por tanto, un método único que pueda ser repetido en otros contextos, $y$ en otras condiciones, cada uno de estos aspectos requiere de la implementación de actividades distintas de enseñanza.

De acuerdo a lo reseñado por Díaz y Hernández (2002), el uso de estrategias metodológicas pertinentes a diferentes momentos del proceso de enseñanza implica la consideración de algunas características esenciales, entre ellas: a) Las características generales del educando, sus conocimientos previos, sus factores motivacionales, su nivel de desarrollo cognitivo, los factores motivacionales, b) La vigilancia permanente del proceso de enseñanza, lo cual tiene que ver con la advertencia continua y oportuna de los logros de aprendizaje, el progreso de la actividad y las estrategias empleadas, c) La intencionalidad que se espera conseguir y las actividades cognitivas y pedagógicas que el educando tiene que hacer para conseguir dicha intencionalidad, y d) Tipo de dominio del conocimiento en general y del contenido curricular que se va a abordar.

Cada una de las características señaladas por los autores se toma en consideración para seleccionar las estrategias más idóneas con las condiciones en que tenga lugar el acto educativo. Estas además pueden interactuar entre sí con la finalidad de que el actor enseñante pueda, no solo elegirlas si no también, el modo en que pueda aplicarlas.

Las consideraciones de estas características también sirven de base para establecer las estrategias pertinentes que sean cónsonas con otras modalidades en el proceso de enseñanza, dado que, además de la modalidad presencial, existen otras en donde el acto educativo se gesta por la asistencia computacional, por señalar algunas en donde las estrategias a utilizar deberán variar y por lo tanto la efectividad y potencialidad de las mismas podrían verse disminuidas por efecto de la poca estimación que se tenga sobre los factores influyentes en el uso de estrategias.

En el ámbito educativo se llevan a cabo muchas estrategias metodológicas capaces de apoyar la promoción de los diferentes aprendizajes generados en el escenario escolar, estas son: 1. Objetivos, los cuales son enunciados que establecen condiciones, tipo de actividad y forma de evaluación del aprendizaje del estudiante; 2. Resúmenes, los cuales consisten en síntesis y abstracciones de la información relevante de un contenido que puede ser oral o escrito; 3. Organizador previo, cuyas informaciones de tipo introductorio $y$ contextual, implica un conector entre la información previa y la nueva; 4. Ilustraciones, estas tienen que ver con representaciones visuales de objetos o situaciones sobre una teoría o tema específico. Estas involucran el uso de fotografías, dibujos, dramatizaciones, etc.; 5. Organizadores gráficos, que son representaciones visuales de conceptos, explicaciones o patrones de información, entre estas se hallan los cuadros sinópticos; 6. Analogías, las cuales son proposiciones que ayudan a indicar que una cosa o evento es semejante a otro. Puede ser el primero concreto o familiar y el segundo, desconocido, abstracto o complejo; 7. Preguntas intercaladas, las cuales 
son preguntas insertadas en la situación de enseñanza o en un texto. Contribuyen las mismas a mantener la atención y favorecen la práctica, la retención y la obtención de información relevante; 8. Señalizaciones, los cuales se hacen en un texto o en la situación de enseñanza para enfatizar u organizar elementos importantes del contenido que se imparte; 9. Mapas y redes conceptuales, las cuales consisten en representaciones graficas de esquemas de conocimiento, estos indican conceptos, proposiciones y explicaciones; 10. Organizadores textuales, que son organizaciones retoricas de un discurso que contribuyen en la comprensión y el recuerdo.

Las variadas estrategias que señalan los autores, pueden incluirse al inicio, durante o al término del acto educativo. Si se llevan a cabo al inicio entonces las estrategias se clasifican en preinstruccionales, las cuales servirán para alertar e incidir en la activación de conocimientos. Al realizarlas durante el acto educativo, estas se pueden considerar como coinstruccionales, las cuales consistirán en el apoyo de contenidos curriculares del proceso de enseñanza. Además cubren funciones para que el aprendizaje mejore la atención y detecte la información principal. Las estrategias emprendidas al final del acto educativo son consideradas como post instruccionales, y las mismas contribuyen a formar una visión sintética, integradora e incluso crítica del material.

En este sentido, y según Díaz y Hernández (2002) la enseñanza corre a cargo del enseñante como su subordinado, pero al fin y al cabo es una construcción conjunta como producto de los continuos y complejos intercambios con los alumnos y el contexto instruccional (institucional, cultural, etcétera), que a veces toma caminos no necesariamente predefinidos en la planificación. Asimismo, se afirma que en cada aula donde se desarrolla el proceso de enseñanza- aprendizaje, se realiza una construcción conjunta entre enseñante y aprendices únicos e irrepetibles. (p. 140)

La variedad de las estrategias que abordan los autores son clasificadas también atendiendo a las intencionalidades de la praxis educativa que se está llevando a cabo. Para tales fines, muchas de las estrategias citadas constituirán grupos que pertenecerán simultáneamente a una y otra clasificación, dependiendo de los objetivos que se persigan.

\section{MÉTODO}

Esta investigación se enmarcó en un enfoque cuantitativo, presentando un diseño no experimental, de campo, la cual según Palella (2012) es "la recolección de datos se realiza de fuentes primarias, se recolectan los datos donde ocurren los hechos" (p. 88); además de ser de tipo descriptivo. Debido a que la problemática en el circuito $n^{\circ} 4$ del municipio Los Guayos en el estado Carabobo, está enfocada en hacer un estudio descriptivo sobre las estrategias metodológicas usadas para la enseñanza de la Ley de Ampere a la luz del enfoque constructivista de Díaz y Hernández. Se realizó un estudio con estas características debido a que se necesita buscar una solución al problema sin hacer ninguna modificación sobre las variables que se presentaron.

Para la ejecución de esta investigación se hizo uso de un cuestionario para ser aplicado a un grupo de docentes conformado por profesores de física del nivel de secundaria. Según Tamayo y Tamayo, "la población se define como la totalidad de fenómenos a estudiar, donde la unidad de población posee una característica común la cual se estudia y da origen a los datos de la investigación". (p.714), en este estudio se tomó como 
población un total de 12 docentes de física pertenecientes al circuito número cuatro del Municipio los Guayo del estado Carabobo, conformado por la Escuela Técnica Francisco de Miranda, Liceo Bolivariano Manuela Sáenz, Unidad Educativa Luis Beltrán y Unidad Educativa. Manuel Cedeño; donde se seleccionó una muestra conformada por siete de los docentes del área.

El instrumento empleado fue un cuestionario con 19 ítems, ordenados según el momento de enseñanza enfocados en Díaz y Hernández, los cuales son: el preinstruccional, el coinstruccional y postinstruccional. El mismo estuvo enfocado en una escala de Likert, por ser un instrumento de respuestas policotómicas.

Este instrumento fue previamente elaborado por Colmenares y Henríquez (2011), en el trabajo titulado: "Estrategias metodológicas utilizadas por los docentes para la enseñanza del contenido Leyes de Newton, basada en el enfoque constructivista de Díaz y Hernández en el liceo Bolivariano Manuel Antonio Malpica, ubicado en el Municipio Naguanagua del estado Carabobo"; pues el mismo ya había sido validado y calculado el grado de confiabilidad. (Alfa de Cronbach, de 0.70)

\section{RESULTADOS Y DISCUSIÓN}

En el proceso en que ha sido sometido este trabajo se han logrado excelentes resultados, los cuales han dejado un amplio conocimiento acerca de las estrategias que utilizan los docentes al enseñar contenidos de física como Ley de Ampere. Al repasar punto por punto cada tema tratado en él, se ha llegado a la conclusión de que hoy en día existen una serie de estrategias metodológicas que los docentes pueden implementar para hacer llegar los contenidos a los estudiantes de manera efectiva. Sin embargo, no siempre se implementan de manera adecuada.

El presente trabajo de investigación tuvo por objeto de estudio la descripción de las estrategias metodológicas que utilizan los docentes al enseñar la ley de Ampere, haciendo referencia a las estrategias docentes de Díaz y Hernández (2002) y a la teoría del aprendizaje significativo del psicólogo Ausubel (2006); los resultados obtenidos dan a conocer cómo los docentes dan un inicio adecuado a cada clase, trabajando con los conocimientos previos que ya posee el estudiante, durante el desarrollo de la misma, lo realizan basándose en ilustraciones, esquemas y por último pero no el menos importante, el cierre del momento pedagógico, donde sintetizan la información suministrada al momento.

Para el análisis por dimensiones se consiguió, en la dimensión preinstruccional se obtuvo un 57\% de los docentes del circuito $\mathrm{N}^{\circ}$ 4 del municipio Los Guayos que implementan estrategias como organizadores previos $y$ lluvia de ideas al momento del inicio de la clase; para esta dimensión, más de la mitad de la población docente enuncia los objetivos, promueve el dialogo interactivo y genera preguntas al iniciar una clase de física.

Por otra parte, en la dimensión coinstruccional se obtuvo un $42 \%$ de los docentes realizan estrategias como gráficos e ilustraciones durante el desarrollo de la clase; en esta dimensión, menos de la mitad de la población docente ilustra, clasifica la información, elabora gráficos y coordenadas al momento del desarrollo de una clase de física.

En la dimensión postinstruccinal se alcanzó un 36\% de los docentes efectúan estrategias como resúmenes y mapas conceptuales al momento del cierre de la clase. Para esta última dimensión, un muy bajo 
número de docentes cumple con los indicadores establecidos al cerrar una clase de física, por lo que se requiere de la incidencia del uso de estas estrategias docentes sugeridas por Díaz y Hernández, para una formación académica, ya que son estos momentos pedagógicos los que organizan adecuadamente una clase.

En síntesis, se puede decir que menos de la mitad de la población docente que se encuentra enmarcada en el circuito $\mathrm{N}^{\circ} 4$ del Municipio Los Guayos del estado Carabobo implementa las estrategias docentes sugeridas por los pedagogos Díaz y Hernández. A su vez, cabe destacar que estos docentes no suelen elaborar experimentaciones ni representaciones de los fenómenos físicos que logran en el estudiante un aprendizaje significativo, permitiéndoles partir de los conocimientos previos que ya poseen, para interceptarlos con los conocimientos nuevos a adquirir y construir nuevos temas.

\section{CONCLUSIONES}

En virtud de los resultados obtenidos se pudo concluir, en cuanto a la dimensión preinstruccional, las estrategias empleadas al inicio de toda actividad pedagógica, fueron: discusión guiada, actividades generadoras, señalización de objetivos centrales, diálogo interactivo. En este sentido se recomienda a los educadores incidir más en la variabilidad y alternancia de estas estrategias con el objeto de dinamizar el conjunto de esquemas y contenidos propios de la estructura cognoscitiva de los estudiantes a fin de que los mismos participen más en el desarrollo de las sesiones de clase.

En esta dimensión se busca principalmente que los estudiantes se interesen en el contenido que se intenta abordar; para ello, se emplean estrategias que activen el interés y la motivación, por lo cual se recomienda la aplicación de actividades experimentales en donde se pongan de manifiesto los fenómenos físicos involucrados con el contenido, a fin de que los estudiantes puedan enlazar sus ideas y conocimientos preestablecidos.

En este orden de ideas, las estrategias anteriormente señaladas, en lo que respecta al contenido de Ley de Ampere, tienen la función de activar los conocimientos previos de los estudiantes, dado que muchos de ellos se han apropiado del contenido en su interacción con los aspectos empíricos vinculados al electromagnetismo.

En la dimensión coinstruccional se establecen estrategias metodológicas que tienen que ver con: ilustraciones, clasificación de la información, imágenes, semejanzas y relaciones, gráficos; donde los contenidos vinculados con el electromagnetismo implican relaciones numéricas y vectoriales. Sobre este particular, se establecen ecuaciones y fórmulas que son empleadas para la aplicación de las teorías comprendidas. Debido a esto, y dada la importancia de la eficiente apropiación de estos temas por parte de los estudiantes se recomienda el uso variado de algunas de las estrategias anteriormente señaladas, con el fin de que los aprendices puedan valerse de las ilustraciones e imágenes para la adquisición de contenidos.

La clasificación de información tiene como objetivo la organización de contenidos para que el educando pueda comprender la importancia y alcance de los temas discutidos. En este sentido, cabe destacar que las ecuaciones y formulas empleadas para la aplicación de las teorías desarrolladas se pueden apreciar en detalles.

De acuerdo a los resultados obtenidos en relación a dimensión postinstruccional, se puede apreciar que el uso de estas estrategias no son 
muy favorecidas por la aplicación didáctica de los agentes de enseñanza, por lo que se recomienda el uso de las mismas en el abordaje de los contenidos de física ya referidos.

Estas estrategias tienen que ver con la organización de resúmenes y síntesis de información, los cuales podrían ayudar a los estudiantes al manejo eficaz de muchos de los contenidos abstractos de física, dado que le permitiría al educando ponderar la información más valedera. En síntesis, se recomienda a la población docente incentivar a los estudiantes al estudio de los contenidos de física haciendo uso de experimentaciones y representaciones de fenómenos físicos.

\section{REFERENCIAS}

Ausubel, D. (2006). Teoría del aprendizaje significativo en la perspectiva de la psicología cognitiva. Barcelona: Octaedro

Bunge M., (1989). La investigación científica: su estrategia y su filosofía. Barcelona: Ariel S. A

Colmenares y Henríquez (2011). Estrategias metodológicas utilizadas por los docentes para la enseñanza del contenido Leyes de Newton, basada en el enfoque constructivista de Díazy Hernández en el liceo Bolivariano Manuel Antonio Malpica, ubicado en el Municipio Naguanagua del estado Carabobo (Trabajo de investigación). Universidad de Carabobo, Venezuela

Díaz, F. y Hernández, G. (2002). Estrategias docentes para un aprendizaje significativo (3 $3^{\underline{a}}$ ed.). México, D.F., México: McGraw-Hill Interamericana

Méndez, F. y Villavicencio, M. (2017). Enseñanza del electromagnetismo a través de aplicaciones experimentales. Universidad Nacional Autónoma de México. Recuperado de https://dialnet.unirioja.es/servlet/articulo ?codigo $=6353422$

Palella S. y Pestana, M. (2012). Metodología de la investigación cuantitativa. Caracas: Editorial Pedagógica Venezolana

Tamayo, y Tamayo, M. (1992). El proceso de la investigación científica. México: Limusa

Universidad Pedagógica Experimental Libertador. (2002). Manual de trabajos de grado de especialización y maestría y tesis doctorales. Caracas: FEDUPEL 